\title{
On the nature of EIT waves, EUV dimmings and their link to CMEs`
}

\author{
A. N. Zhukov ${ }^{1,2}$ and F. Auchère ${ }^{3}$ \\ 1 Royal Observatory of Belgium, Avenue Circulaire 3, 1180 Brussels, Belgium \\ e-mail: Andrei.Zhukov@oma.be \\ 2 Skobeltsyn Institute of Nuclear Physics, Moscow State University, 119992 Moscow, Russia \\ 3 Institut d'Astrophysique Spatiale, Université Paris XI, Bât. 121, 91405 Orsay Cedex, France
}

Received 27 February 2004 / Accepted 5 July 2004

\begin{abstract}
EIT waves and extreme-ultraviolet (EUV) dimmings attract particular attention as they frequently accompany Coronal Mass Ejections (CMEs). We present several examples of EIT waves and EUV dimmings with particular morphologies previously unreported in the literature. We report for the first time an EIT wave in the Fe XV (284 ̊) bandpass of the SOHO/EIT instrument. The observations of this event confirm previous results that an EIT wave is a purely coronal phenomenon that does not propagate in the transition region plasma. Two EIT wave events initiated close to the solar limb are investigated, thus permitting us to see simultaneously the wave and the magnetic configuration of the CME. These observations suggest that EIT wave can be regarded as a bimodal phenomenon. The wave mode represents a wave-like propagating disturbance. Its characteristic features are propagation of a bright front to large distances from dimming sites and quasi-circular appearance. The eruptive mode is the propagation of a dimming and of an EIT wave as a result of successive opening of magnetic field lines during the CME lift-off. It can be identified by noting the expansion of a dimming and the appearance of another dimming ahead of a bright front. We reveal the temperature structure of the EUV dimmings that appeared after the classical EIT wave event on May 12, 1997, using differential emission measure (DEM) maps obtained through the analysis of images in four EIT bandpasses. The part of the CME mass contained in the low corona observed by the EIT is estimated to be about $10^{15} \mathrm{~g}$. It appears that around 50\% of this total CME mass in the low corona is contained outside of transient coronal holes. It is shown that at present it is difficult to reconcile all the observational facts into a coherent physical model. In particular, the physical nature of the wave mode of EIT waves remains elusive.
\end{abstract}

Key words. Sun: corona - Sun: coronal mass ejections (CMEs)

\section{Introduction}

Coronal Mass Ejections (CMEs) are probably the most spectacular phenomena observed on the Sun. Their study is also important for solar-terrestrial relations as some CMEs may be directed towards the Earth and - with a suitable magnetic field orientation - produce geomagnetic storms. In this regard the problem of CME initiation deserves particular attention as its solution may ultimately lead to the prediction of CME occurences.

The origin of CMEs cannot be traced by coronagraphs, as the occulting disk obscures a direct view of the initiation site. This is why observations of the low corona are necessary. The Extreme-ultraviolet Imaging Telescope (EIT, see Delaboudinière et al. 1995) onboard the Solar and Heliospheric Observatory (SOHO) with its continuous $24 \mathrm{~h}$ per day full disk coverage in four extreme-ultraviolet (EUV) bandpasses is well

$\star$ Movies are available in electronic form at http://www. edpsciences.org suited for the detection of CME signatures in the low corona (and also in the transition region). Among others, the observations made by EIT are used to obtain information about the still-enigmatic CME initiation mechanism.

After the discovery of the so-called "EIT wave" phenomenon (Thompson et al. 1998) it was soon suggested that these waves are strongly associated with CMEs (Plunkett et al. 1998). Later, the statistical study of 173 EIT wave events in March 1997-June 1998 by Biesecker et al. (2002) confirmed this association. Most often an EIT wave appears as a propagating bright front observed in the $195 \AA$ bandpass of the EIT. It sometimes propagates nearly isotropically and often globally, i.e. on a large part of the visible solar disc, stopping at the boundaries of coronal holes (Thompson et al. 1998). EIT waves also avoid active regions (Thompson et al. 1999), although they may trigger transverse oscillations of active region loops (Wills-Davey \& Thompson 1999). The propagation speed of EIT waves is typically around $250 \mathrm{~km} \mathrm{~s}^{-1}$ (Thompson et al. 1998, 1999), although non-isotropic propagation and 
inhomogeneity of the front sometimes suggest that the speed could be up to $800 \mathrm{~km} \mathrm{~s}^{-1}$ (Wills-Davey \& Thompson 1999). However, usually EIT wave speeds do not exceed $400 \mathrm{~km} \mathrm{~s}^{-1}$.

Behind an EIT wave, a dimming is usually observed; sometimes it also propagates following the wave front. Often this dimming appears as a transient coronal hole $(\mathrm{TCH})-$ its appearance resembles that of polar coronal holes. Sometimes dimmings (and TCHs in particular) appear in pairs, suggesting that such a double (twin) dimming represents the footpoints of an ejected interplanetary flux rope (Webb et al. 2000). However, this interpretation was contested by Kahler \& Hudson (2001). In particular, they argue that TCH lifetimes (less than $48 \mathrm{~h}$ in nearly all cases) are shorter than travel times of the flux rope CME to the Earth (typically 3-5 days). Nevertheless it appears possible that TCHs can represent the footpoints of the flux rope until their disappearance and eventual flux rope disconnection from the Sun.

Thompson et al. (2000a) found that large but weak dimming areas may extend to large distances from the TCHs. These extended dimmings correspond well to the CME locations registered by the SOHO/LASCO coronagraph and projected back onto the solar disc.

Thompson et al. (1998) showed that TCHs exhibit approximately the same pattern in the three coronal bandpasses of the EIT. They thus conclude that dimmings are rather due to the evacuation of mass during the CME than to changes of plasma temperature. Harra \& Sterling (2001) used the SOHO Coronal Diagnostic Spectrometer (CDS) data in the O V line (629 $\AA$ ) to analyze an eruption on July 19, 1999. They detected blueshifts in the dimming region corresponding to outflow velocities up to $80 \mathrm{~km} \mathrm{~s}^{-1}$ and concluded that dimmings are indeed due to the ejection of mass.

Delannée (2000) reported several examples of events with dimmings occurring not only behind, but also ahead of the EIT wave bright front. Moreover, in these events EIT waves did not propagate isotropically, but rather in several directions corresponding to large-scale loops connecting the eruptive active region (AR) with other active regions and strong concentrations of the magnetic field, sometimes in the opposite hemisphere. On the basis of these observations Delannée (2000) proposed that EIT waves are not "real" magnetohydrodynamic (MHD) waves. When the low-lying active region loops rise and erupt (thus forming a characteristic double dimming pattern), the overlying large-scale loops rise and erupt as well. The dimming ahead of the bright front is situated at the distant footpoints of these large-scale loops overlying the erupting active region. The bright front appears due to the plasma compression in the process of successive opening of magnetic field lines during the CME lift-off (Delannée \& Aulanier 1999; Chen et al. 2002). A comprehensive diagram illustrating this idea can be found in the work by Wang et al. (2002).

In the present paper we report several interesting and particular examples of EIT waves that may shed additional light on the CME onset problem. EIT waves and similar phenomena in different spectral domains are discussed in Sect. 2. We report for the first time (to our knowledge) an example of an EIT wave in the Fe XV (284 $\AA$ ) bandpass of the EIT. In Sect. 3 we describe two EIT wave events initiated close to the solar limb.
On the basis of both their on-disc and off-disc observations we propose the concept of EIT wave bimodality. In Sect. 4 we estimate the CME mass ejected from the low corona observed by the EIT. In Sect. 5 we summarize our results and point out questions that are still unresolved.

\section{EIT waves observed in different parts of the solar spectrum}

During most of the time EIT makes observations in the coronal Fe XII (195 ^) bandpass. Thus EIT waves and EUV dimmings have been primarily observed in the $195 \AA$ bandpass. A natural question is: can they be also observed in other spectral domains?

Almost four decades before the first observation of an EIT wave a similar phenomenon was discovered in the chromosphere - a Moreton wave (Moreton 1960; Moreton \& Ramsey 1960). It represents a dark arc-shaped front propagating out from the flare site observed in the center or wings of the $\mathrm{H} \alpha$ line. Most often it is seen up to distances of only $5 \times 10^{5} \mathrm{~km}$ (along the photospheric surface) from the flare site (Moreton \& Ramsey 1960), in contrast to EIT waves sometimes propagating globally over the whole visible solar disc (Thompson et al. 1998, 1999). The main difference between the two types of waves appears to be that the speeds of Moreton waves are usually about $800-1000 \mathrm{~km} \mathrm{~s}^{-1}$ and even larger (Moreton \& Ramsey 1960), which is significantly higher than the speeds of EIT waves (usually around $250 \mathrm{~km} \mathrm{~s}^{-1}$ ). Biesecker et al. (2002) proposed that there are two types of EIT waves: waves with a diffuse front, travelling slowly (a typical example is the classical event of May 12, 1997 reported by Thompson et al. 1998) and waves with a sharp bright front, travelling faster (such as the typical example event of September 24, 1997 reported by Thompson et al. 2000b). It is argued that EIT waves of the second type (they constitute only 7\% of the total number of EIT waves from March 1997 to June 1998) are the coronal counterparts of Moreton waves (Biesecker et al. 2002). We note, however, that during the September 24, 1997 event the EIT wave had a sharp front only in the first frame, then the front became diffuse.

Only a few studies of simultaneous observations of EIT and Moreton waves were performed. The main problems for this type of investigation are, on one hand, the rarity of Moreton wave observations and, on the other hand, the undersampling of the wave propagation by the EIT in time (the typical cadence of observations is only around $12 \mathrm{~min}$ and thus EIT registers only 2-3 wavefronts). Thompson et al. (2000b) have reported that the EIT wave front was cospatial with the area of propagation of the Moreton wave for the event of September 24, 1997.

Moreton waves usually decelerate down to speeds in the range of 200-650 $\mathrm{km} \mathrm{s}^{-1}$ (Warmuth et al. 2002, 2004a). It was shown (Warmuth et al. 2001, 2002, 2004a) that although EIT and Moreton wave fronts have different speeds, they lie on the same kinematic curves (if the curves are approximated by a parabolic fit). Warmuth et al. (2001, 2004a,b) conclude that the low speed of an EIT wave is explained by the deceleration of a Moreton wave. However, Eto et al. (2002) have described an event where the oscillations of a distant filament (presumably 
triggered by the arrival of the Moreton wave) had started earlier than the arrival of the EIT wave. Therefore, they approximated the kinematic curves for the Moreton and EIT waves by two different linear fits and argued that these two waves represent different phenomena.

Vršnak et al. (2002) reported three examples of propagating waves observed in the $10830 \AA$ line of He I. The waves seemed to be strongly associated with the Moreton waves observed simultaneously. An EIT wave has been registered in only one of the events (November 25, 2000) and its speed was $285 \mathrm{~km} \mathrm{~s}^{-1}$. It is again lower than both the Moreton wave speed $\left(836 \mathrm{~km} \mathrm{~s}^{-1}\right.$ at the beginning of the event) and He I wave speeds ( $385 \mathrm{~km} \mathrm{~s}^{-1}$ for this event and $450-600 \mathrm{~km} \mathrm{~s}^{-1}$ for other events). Due to the complicated mechanism of the He I line formation (it reflects physical conditions in the upper chromosphere, transition region and low corona), the interpretation of these observations is difficult. Gilbert et al. (2004) described two waves observed simultaneously in He I and by the EIT and argued that the He I wave is not an MHD wave but an "imprint" of the EIT wave propagating in the corona. Warmuth et al. (2004a) mentioned also the existence of the Moreton wave counterpart observed in the $17 \mathrm{GHz}$ radioheliograms.

It is sometimes stated that Moreton waves are flareassociated waves and all the wave-like phenomena described above (EIT waves, Moreton waves, waves in He I) are named "flare waves" (e.g. Vršnak et al. 2002; Warmuth et al. 2003, 2004a,b). We note, however, that the association of Moreton waves with flares results mostly from the fact that at the time of the Moreton wave discovery (1960s) the CME phenomenon was not yet known (the first CMEs were observed in the 1970s). A statistical study of Moreton waves associated with other phenomena in the solar atmosphere like flares or CMEs has not yet been performed, in part because there are just a few observations of Moreton waves. It is safe to suggest that Moreton waves also could be CME-associated.

As the same physical mechanisms have been proposed to explain Moreton and EIT waves, it is appropriate to briefly address here the question of their modeling. It appears that for the EIT and Moreton wave models a crucial assumption is the value of the coronal plasma parameter $\beta=\frac{p}{B^{2} / 8 \pi}$ (the ratio of plasma pressure $p$ and magnetic pressure $B^{2} / 8 \pi$ ). According to the model of Moreton waves developed by Uchida (1968, 1974) and Uchida et al. (1973) the initial pressure pulse (representing, for example, a flare) launches a quasi-spherical fast mode wave propagating in the low-beta $(\beta<1)$ corona at a speed close to the plasma fast magnetosonic speed (of the order of $1000 \mathrm{~km} \mathrm{~s}^{-1}$ ). This wave may or may not steepen to form a shock. Its skirt sweeps the surface of the chromosphere thereby producing the Moreton wave.

The first models of EIT waves - the ray-tracing method by Wang (2000) following Uchida's model or full 3D timedependent MHD simulations by Wu et al. (2001) - also interpreted them as fast magnetosonic waves. These models describe well the quasi-circular shape of EIT waves and a number of other important properties. It should be stressed, however, that to account for the low EIT wave velocities both models (Wang 2000; Wu et al. 2001) had to adopt values of the coronal magnetic field resulting in high values of the plasma parameter beta $(\beta>1)$. This does not seem to be realistic in the low corona: as Wu et al. (2001) point out, the Wilcox Solar Observatory photospheric magnetograms that they used for the coronal field calculations have a low spatial resolution and cannot measure strong magnetic fields in sub-arcsecond concentrations. The attempt to associate Moreton and EIT waves (Warmuth et al. 2001, 2004a,b) is also based on high values of $\beta$. As noted above, low $(\beta<1)$ values of the coronal beta give a fast mode speed around $1000 \mathrm{~km} \mathrm{~s}^{-1}$ (Uchida et al. 1973). The EIT wave speeds are close to the slow mode speed along the magnetic field in a low- $\beta$ plasma, but EIT waves often propagate quite isotropically.

A way to resolve this discrepancy has been proposed by Chen et al. (2002) who performed 2.5D MHD simulations of an EIT wave propagation. In contrast to all the earlier models, the wave is launched after the start of the flux rope rise (simulating a CME) in a low- $\beta$ corona and thus represents a piston-driven wave. This model describes the Moreton wave as the piston-driven fast mode shock propagating at a speed around $800 \mathrm{~km} \mathrm{~s}^{-1}$. The EIT wave appears as a result of successive opening of the field lines (cf. Delannée 2000; Delannée \& Aulanier 1999) and thus is not a real MHD wave. The speed of this wave-like disturbance agrees rather well with the observed values of the EIT wave speed (around $250 \mathrm{~km} \mathrm{~s}^{-1}$ ). However, the simulations by Chen et al. (2002) are performed in a simplified 2.5D geometry, so it is hard to envision how an EIT wave front can be almost circular as in the May 12, 1997 event described by Thompson et al. (1998).

EIT waves were registered in the $171 \AA$ bandpass as well, and the most remarkable example observed by the TRACE (the Transition Region and Coronal Explorer, see Handy et al. 1999) is reported by Wills-Davey \& Thompson (1999). The observations have been made almost simultaneously in three TRACE bandpasses: $195 \AA, 171 \AA$ (corona) and in the Ly $\alpha(1216 \AA$ ) chromospheric bandpass. The propagation of the wave has been registered in both $195 \AA$ and $171 \AA$ (it was weaker in the latter than in the former), but not in the Ly $\alpha$. Low-lying bright points have not been affected by the propagation of the wave. As the $171 \AA$ bandpass of the TRACE (as well as the one of the EIT) contains strong contribution from the relatively cool upper transition region (TR) plasma, Wills-Davey \& Thompson (1999) conclude that the EIT wave is a coronal phenomenon and does not propagate in the transition region.

A phenomenon similar to EIT waves is sometimes registered in the soft X-rays (SXR). However, Yohkoh/SXT and GOES-12/SXI telescopes did not observe many events of this kind. Strong scattered light during flares is a major problem preventing efficient detection of weak EIT wave fronts in the SXR (Hudson et al. 2003). The speeds reported for several examples observed by the SXT (see e.g. Khan \& Aurass 2002; Narukage et al. 2002) were of the order of $500 \mathrm{~km} \mathrm{~s}^{-1}$. In the event described by Narukage et al. (2002) the Moreton wave was observed simultaneously and propagated with similar speed. Taking $\beta$ around 0.5 gives the fast mode wave speeds around $500 \mathrm{~km} \mathrm{~s}^{-1}$ (Narukage et al. 2002). On the other hand, according to the preliminary results of the EIT waves search in the SXI data (Biesecker 2003) the wave speeds in the SXR are 
between $50 \mathrm{~km} \mathrm{~s}^{-1}$ and $350 \mathrm{~km} \mathrm{~s}^{-1}$. These values agree with typical EIT wave speeds.

Here we report for the first time (to our knowledge) an EIT wave observed in the Fe XV (284 $\AA)$ bandpass of the EIT during a "CME Watch 284" series (with cadence around 13 min). The eruption occurred on February 9, 1999 originating in NOAA AR 8453 and was associated with a partial halo CME first seen by the LASCO C2 coronagraph at 05:33 UT. The development of the event is shown in Fig. 1. The first frame shows the unperturbed Sun at 04:24 UT - right before the event. The bright front around the eruption site is evident in the running difference image shown in the second panel of Fig. 1 (04:49-04:36 UT); the dimming (marked as "d1" in the third panel of Fig. 1) is located behind the front. On the next image (05:02-04:49 UT) the front moved further (at a speed around $50 \mathrm{~km} \mathrm{~s}^{-1}$ ) and the second dimming ("d2" in Fig. 1) appeared ahead of it, apparently tracing the magnetic field lines connecting erupting NOAA AR 8453 with NOAA AR 8455 to the east of it. The dimming " $\mathrm{d} 2$ " also propagates, and there are some weak brightenings possibly indicating a bright interrupted front ahead of it. The third dimming ("d3" in Fig. 1) becomes clearly prominent at 05:36 UT close to the northwestern limb; it also propagates and evolves. There are also indications of another large dimming ("d4" in Fig. 1) to the south-west of NOAA AR 8453, but it is weaker. All these dimmings are accompanied by weak brightenings ahead of them indicating the front of the EIT wave.

Overall, the EIT wave and EUV dimming configuration appears to be very complicated in this event. Wave fronts ahead of dimmings "d2" and "d3" propagate at a speed around $300 \mathrm{~km} \mathrm{~s}^{-1}$; this value is however approximative, as the fronts are very weak and their positions are thus difficult to mark. We stress that the fronts and especially the dimmings propagate approximately in three directions: to the east towards the NOAA AR 8455 (d2), to the north-west (d3) and, possibly, to the south-west (d4). Therefore, this event belongs to the type described by Delannée (2000) with dimmings tracing the opening of magnetic field lines.

It is interesting to note that the dimming " $\mathrm{d} 3$ " appearing to the north-west of the NOAA AR 8453 close to the limb does not show a clear propagation (unlike others). It does not exist at 05:11 UT, and it is clearly seen at 05:36 UT. In the difference image 05:25-05:11 UT one can see some weak dimming between the NOAA AR 8453 and the dimming "d3" at 05:36 UT. It appears that this weak dimming traces the propagation of the dimming "d3" from the eruption site, but in this case it is almost invisible in the region "A" (see the first panel of Fig. 1).

To understand the reason for such a behaviour we inspected plain EIT images in the $284 \AA$ bandpass; a typical one (taken at 04:24 UT) is presented in the first panel of Fig. 1. It is evident that the region " $\mathrm{A}$ " is weaker than the surrounding corona and is dominated by the network structure. This strongly suggests that in this part of the Sun the emission in the $284 \AA$ bandpass of the EIT is dominated not by the coronal Fe XV line at $284.160 \AA$ (peak formation temperature $\log T_{\max }=6.3$ ) but rather by the cooler upper transition region lines - Si VII at $275.353 \AA\left(\log T_{\max }=5.8\right)$, Si VIII at $277.057 \AA\left(\log T_{\max }=\right.$ 5.9), Mg VII at $278.393 \AA\left(\log T_{\max }=5.8\right)$. A similar behaviour of the $284 \AA$ bandpass of the EIT has been reported by Del Zanna et al. (2003) for polar plumes.

Therefore, the CME observations in the $284 \AA$ bandpass reported in this section allow us to conclude that at least in this event the EIT wave does not propagate in the upper transition region, but only in the corona. The same result was obtained for an EIT wave observed in the $171 \AA$ bandpass (Wills-Davey \& Thompson 1999).

The on-disc CME signatures in the He II $304 \AA$ bandpass of the EIT have not yet been studied in detail. Images of the "CME Watch 304" sequence (with cadence around $12 \mathrm{~min}$ ) are not taken frequently. Delannée (1999) did not find EIT waves in the "CME Watch 304" dataset over 6 days in March April 1998. However, propagating disturbances somewhat similar to EIT waves are sometimes observed in the $304 \AA$ bandpass. It is not clear though how to interprete these observations. One of the difficulties here is that except for the cool He II line (formed at $303.78 \AA$ in the low TR with $\log T_{\max }=4.9$ ) the $304 \AA$ bandpass also contains the coronal Si XI line at $303.324 \AA\left(\log T_{\max }=6.2\right)$. Thus it is often not clear if propagating disturbances are of TR or coronal nature.

On the contrary, dimmings in the $304 \AA$ bandpass have been reported (Chertok \& Grechnev 2003). However, synoptic images with low cadence $-6 \mathrm{~h}-$ were used in this study. In the classical EIT wave event of May 12, 1997 the transient coronal holes were not seen in the $304 \AA$ bandpass, although some dimming was evident (see Sect. 4 and Fig. 4 below).

\section{Bimodality of EIT waves}

EIT waves are often regarded as real waves in spite of the fact that their interpretation as fast-mode MHD waves has been seriously challenged (see Sect. 2). As we pointed out in Sect. 1, however, many EIT waves exhibit morphologies representing rather the successive opening of the magnetic field lines with dimmings caused by the evacuation of plasma upwards along these newly opened field lines. (Topologically these field lines remain close, but in the low corona observed by the EIT they appear open as the closure occurs much higher.) We note that although dimmings are sometimes also seen to be propagating, it still appears premature to claim the existence of propagating dimmings without an EIT wave (bright front) ahead of them. It seems that one can always find some brightening on the frontal edge of a propagating dimming, even if it is weak and not forming a clear continuous front, and argue that the front exists but its amplitude is very close to the detection threshold. Therefore, it appears reasonable to suppose that dimmings caused by eruptions (distinct from thermal dimmings) are always accompanied by an EIT wave, although dimmings seem to be easier to detect. In some occasions a dimming (and bright front ahead of it) can be seen only in a single frame of the EIT image sequence, so the issue about propagation is open. However, it is probable that in such cases the EIT wave propagates as well, but the low temporal cadence (usually around $12 \mathrm{~min}$ ) does not allow to see this propagation.

EIT waves are strongly associated with CMEs (Biesecker et al. 2002), and CMEs indeed involve the opening of the field lines and lifting of matter. The process of magnetic field lines 


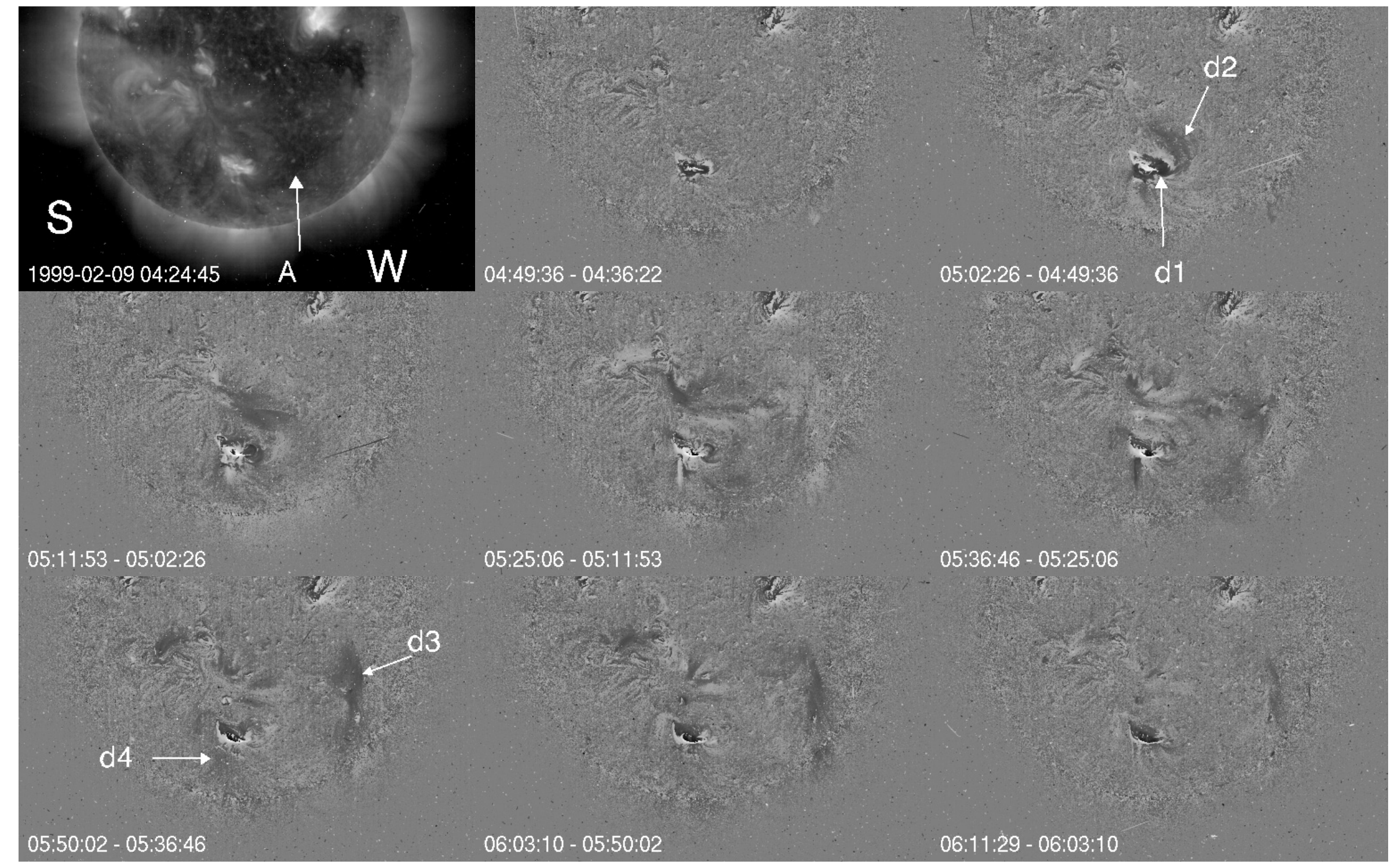

Fig. 1. The initiation of a CME on February 9, 1999 observed by the SOHO/EIT in the Fe XV bandpass (284 $⿱$ ). All the panels are running difference images (with contrast increased) except the first one, which is a plain image. Solar west is at bottom right (as shown in the first panel), south is at bottom left corner of images. All times are UT. 


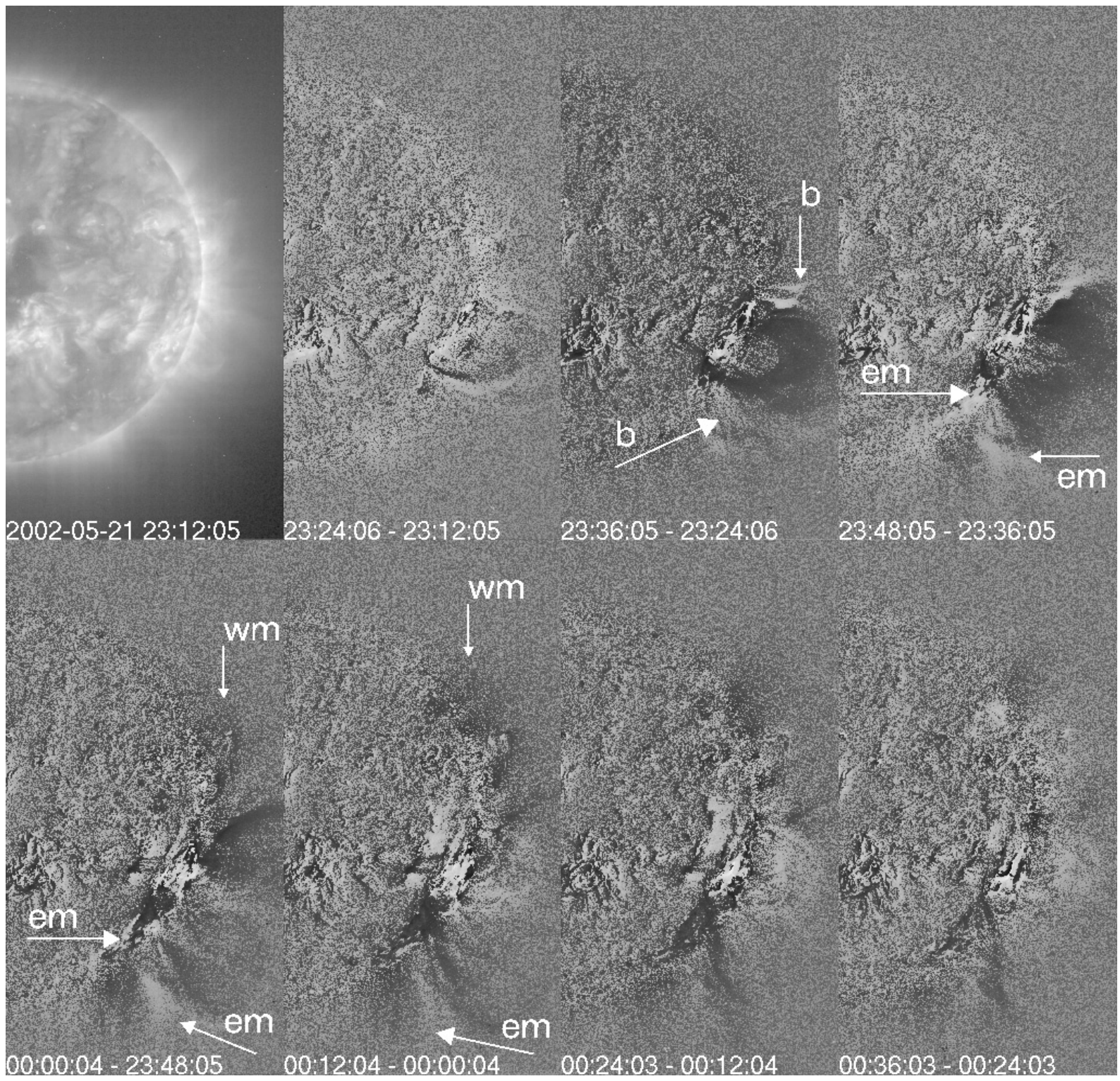

Fig. 2. The initiation of a CME on May 21-22, 2002 observed by the SOHO/EIT in the Fe XII bandpass (195 $\AA$ ). All the panels are running difference images (with contrast increased) except the first one, which is a plain image. "b" marks a brightening, "em" and "wm" mark eruptive and wave modes of the EIT wave respectively (see text for explanations). North is on top, west is to the right. All times are UT.

opening is better seen on the limb, but EIT waves are most successfully detected closer to the center of the disc. We describe here two examples of CMEs with source regions situated close enough to the limb to see the changes of their magnetic structure but at the same time far enough from the limb to make the detection of EIT waves possible.

The first CME occurred on May 21-22, 2002 (first appearance in the LASCO C2 field of view at 00:06 UT on May 22) originating in NOAA AR 9948. Figure 2 illustrates the initiation of this CME. The first frame shows the pre-eruption image (23:12 UT); NOAA AR 9948 is situated close to the south-western limb and has an extended (although weak) loop structure off-disc. The first running difference image (23:24-23:12 UT) shows the initial slight changes in the active region which then became drastic in the next frame (23:36-23:24 UT) - a big loop-like dimming appears above the limb as the plasma is apparently ejected upwards. At its northern and southern sides the dimming is accompanied with brightenings (marked as "b" in Fig. 2). These brightenings can represent either compression of loops moving sidewards (with respect to the main loop system of the AR) or the front of the EIT wave seen from the side. The southern brightening is probably of the first type, but we cannot conclude for sure about the northern one.

The big loop-like dimming above the limb has its counterparts on the disc in the form of small twin dimmings (the southern one is better discernible). Let us follow the evolution of this southern dimming first. The next frame (23:48-23:36 UT) shows the expansion of the dimming above the limb with a clear bright front (shown by the arrow and marked as "em" in Fig. 2) ahead of it. This bright front propagates along the limb to the south (compare the running difference images of 23:48-23:36 UT and 00:00-23:48 UT) and probably represents the front of the EIT wave (if we could look at it from an 


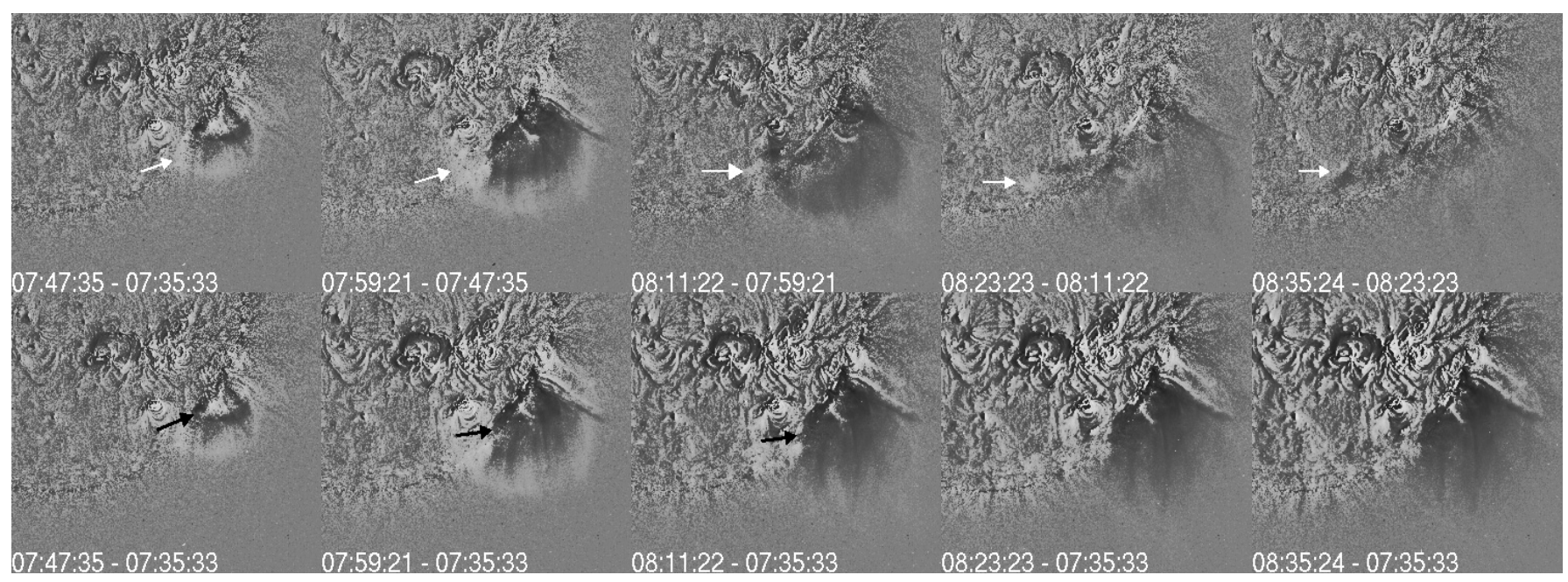

Fig. 3. The initiation of a CME on May 27, 2000 observed by the SOHO/EIT in the Fe XII bandpass (195 Å). Top row: running difference images (previous image of a sequence is subtracted from every image). Bottom row: base difference images (the first image of a sequence taken at 07:35:33 UT is subtracted from all the other images). Solar north on top, west at right. White arrows show the propagation of the EIT wave front on-disc. Black arrows show the propagation of a dimming on-disc. All times are UT.

appropriate position, i.e. from the west in the image plane). The on-disc counterpart of this bright front (also shown by an arrow and marked as "em" in Fig. 2) is less distinct and does not have a continuous appearance. However, its propagation is evident by comparing these two frames. The on-disc dimming behind this bright front also propagates (00:12-00:00 UT). This means that the transient coronal hole is expanding as the field lines more and more distant from the AR become open (see Chen et al. 2002) and the plasma is lifted upwards along them. Another propagating dimming is seen above the limb ahead of the bright front (00:00-23:48 UT and 00:12-00:00 UT). This is the evidence of the overlying field lines opening (cf. Delannée 2000).

On the basis of these observations we conclude that the dimmings propagating to the south represent a typical example of the morphology described by Delannée (2000). It is the propagation of a dimming (and a bright front ahead of it) as a result of successive opening of magnetic field lines with lifting of the coronal plasma along these new quasi-open field lines. Therefore, we call this part of the overall EIT wave event the eruptive mode of the wave (marked as "em" in Fig. 2). Its propagation speed in this case is of the order of $60 \mathrm{~km} \mathrm{~s}^{-1}$ (with similar values on-disc and off-disc; note, however, that the on-disc estimate is subject to uncertainty because the measured points lie very close to the limb). This is slow compared to the classical value of the EIT wave speed $\left(250 \mathrm{~km} \mathrm{~s}^{-1}\right)$. We note also that the expansion of the TCH was seen at least up to 00:48 UT (not shown in Fig. 2), i.e. about an hour after the start of the eruption (23:24 UT) or the evacuation of the most of the CME matter off-disc (23:48 UT). Low velocity and late TCH expansion both indicate the diversity of morphologies of the eruptive mode.

After 23:48 UT there is no clear bright front of the EIT wave propagating to the north of the eruption site. However, the weak dimming (marked as "wm" in Fig. 2) does propagate off-disc (it appears clearly in the difference image between 00:12 and 00:00 UT). This dimming has rather a wave-like appearance - we do not see the motion or opening of the field lines. Therefore, we call this part of the EIT wave a wave mode ("wm" in Fig. 2). It propagated at a speed of around $250 \mathrm{~km} \mathrm{~s}^{-1}$ which agrees well with the classical value. We note, however, that it is nevertheless possible that this propagating dimming represents an eruptive mode. In this case we may not see the motion and opening of the field lines as it happens higher in the corona - outside of the EIT field of view. Additionally, this dimming seems to persist for about an hour (it still could be seen in the difference image 00:36-00:24 UT in Fig. 2). This also indicates that matter erupted from this place.

The existence of the wave mode in the event of May 21-22, 2002 may be doubtful. So, the second event that we are going to describe now displays the wave mode better than the eruptive mode. The CME was first registered in the LASCO C2 field of view at 08:30 UT on May 27, 2000. Figure 3 shows the onset of this CME observed by the EIT in the $195 \AA$ A bandpass. We show both running difference images (top row) and base difference images (bottom row). Running difference images are suitable to show the propagating wave front; base difference images permit us to identify dimmings (places from where the matter erupts). The propagation of the EIT wave bright front on-disc is clearly seen in the running difference images (marked by white arrows). Its positions at the limb correspond rather well to the locations of the semi-circular bright structure off-limb. It is possible that this structure is a projection on an image plane of a 3-dimensional dome-like wave front. The on-disc wave propagates far from the eruption site (08:35-08:23 UT). The absense of clear dimmings there (08:35-07:35 UT) indicates that this bright front represents the wave mode.

The on-disc propagation of a dimming is shown by black arrows in the base difference images in Fig. 3 and most probably signifies the existence of the eruptive mode in this event. The post-eruption arcade seen at 09:12 UT (not shown in Fig. 3) also confirms that the eruption indeed happened. 


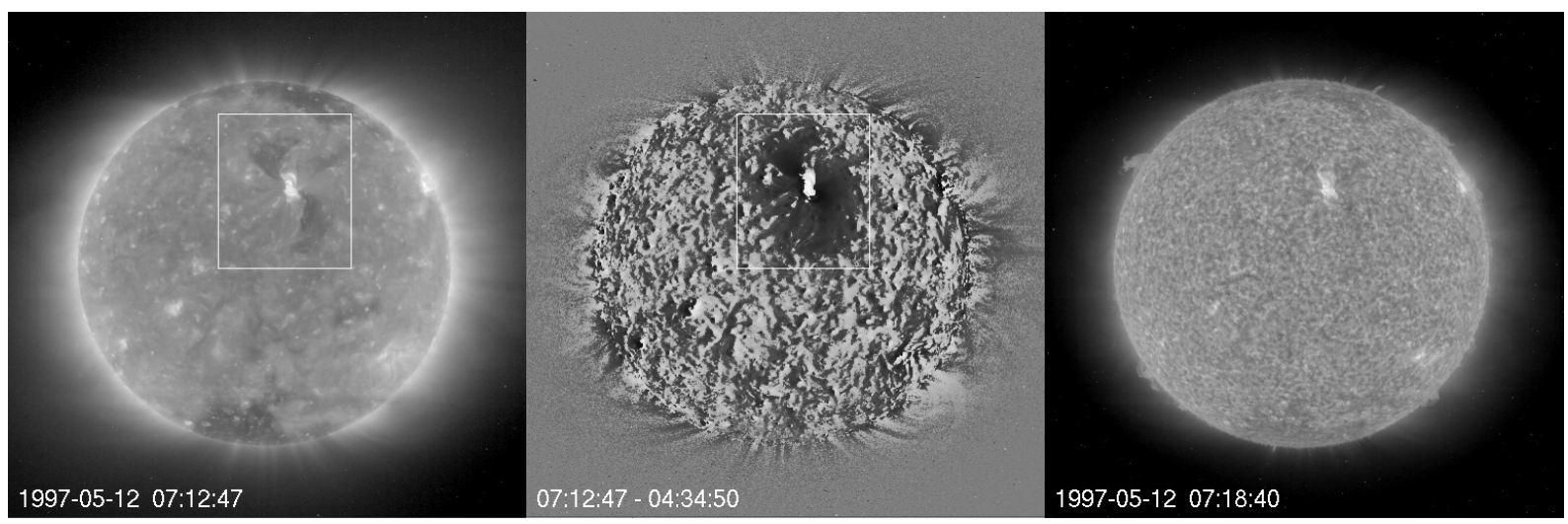

Fig. 4. Left panel: the Sun observed by the SOHO/EIT in the Fe XII bandpass (195 ̊̊) on May 12, 1997 at 07:12:47 UT. A pair of transient coronal holes is seen in the northern hemisphere. Middle panel: a difference of the image in the left panel and the last pre-eruption image (taken at 04:34:50 UT) showing the overall dimming resulting from the material lifting during the CME. The white boxes in the left and middle panels show the area used for the calculation of the erupted mass (see text). Right panel: the Sun observed by the SOHO/EIT in the He II bandpass (304 Å) on May 12, 1997 at 07:18:40 UT. Unlike polar coronal holes, transient coronal holes are not seen, although some weak dimming is evident at their locations inferred from the left panel.

The bright front of the eruptive mode should be situated ahead of the dimming, but in this case it is impossible to discriminate it from the wave mode front. The off-disc bright structure also corresponds to the probable position of the eruptive mode bright front and thus may represent rising loops. We note though that the pre-eruption structures above the limb were very diffuse and did not show loops that could rise and open.

The propagation of the EIT wave to the north seems to stop because it encounters a quasi-open magnetic structure. This structure is pushed aside at the time of the wave arrival as seen in the running difference image at 07:59-07:47 UT and in the base difference image at 07:59-07:35 UT.

It is difficult to distinguish clearly the eruptive mode front for this event, but its existence is exhibited by the propagation of a dimming seen in the base difference images. The existence of the wave mode is evidenced by the absence of pronounced dimmings at the farthest locations reached by the EIT wave (at 08:35 UT). The eruptive mode speed is about $150 \mathrm{~km} \mathrm{~s}^{-1}$, and the wave mode shows deceleration from $300 \mathrm{~km} \mathrm{~s}^{-1}$ to $200 \mathrm{~km} \mathrm{~s}^{-1}$. These values are typical for EIT waves, but as we measured them very close to the solar limb, the accuracy of our estimates may be low.

In this Section we presented the observations of two CME onsets. In both of them we found signatures of the wave mode and of the eruptive mode of EIT waves. The characteristic features of the wave mode are more easily seen in running difference images; they include propagation of a bright front to large distances from dimming sites and sometimes quasi-circular appearance of the on-disc bright front (Thompson et al. 1998). The eruptive mode can be readily identified in the base difference images noting the expansion of a dimming and appearance of another dimming ahead of the bright front. The eruptive mode is better seen in the first example (CME on May 21-22, 2002); the wave mode - in the second one (CME on May 27, 2000). However, the 3-dimensional structure of these two CMEs is still unknown. It is possible that the eruptive mode shown by the opening of the field lines would not be seen as a propagating bright front of the EIT wave. The
STEREO mission (Solar - Terrestrial Relations Observatory) will give more precise information about this issue, by looking at the same eruption from two different positions.

\section{Transient coronal holes and CME mass}

EIT waves often (but not always) propagate over all the visible solar disc, but the CME source region usually has a smaller size. It is supposed (see Sect. 1) that places of matter evacuation from the corona are seen as transient coronal holes (TCHs). Their dimensions are much smaller than the sometimes huge distances travelled by the EIT wave. Additionally, dimmings are often extended to larger areas than TCHs (Thompson et al. 2000a). We have investigated the classical EIT wave event of May 12, 1997 trying to clarify the relation between the region of erupted matter and transient coronal holes.

Figure 4 shows that the pair of TCHs represents only part of the overall dimming, in agreement with the conclusion made by Thompson et al. (2000a). TCHs occupy around $1 / 3$ of the total dimming area. The dimming roughly correponds to the region behind the front of the EIT wave at 05:07 UT, see Fig. 2 in the paper by Thompson et al. (1998). This EIT wave is dominated by the wave mode that propagates almost over the whole solar disc. The eruptive mode probably cannot be distinguished from the wave mode for this event as the EIT cadence was too low (about $17 \mathrm{~min}$ ).

We have estimated the total mass erupted from the low corona (observed by the EIT) and the mass erupted only from the TCHs. Assuming for simplicity a constant density, the emission measure (EM) can be written in zeroth approximation as $E M=n^{2} H$, where $n$ is the particle number density, $H$ is the pressure scale height (we took a typical value of $100 \mathrm{Mm}$ for our calculations). The mass contained in a column of height $H$ and area $S$ is $m=m_{\mathrm{p}} n H S$, where $m_{\mathrm{p}}$ is the proton mass (the corona is assumed to be fully ionized hydrogen plasma). So, we can calculate the mass that erupted from the TCHs and from the overall dimming by subtracting the post-eruption mass from the pre-eruption one for each of the regions. 
We have to calculate the emission measure first. As the bandpass ratio method often gives incorrect results (see e.g. Schmelz et al. 2003), we have used the EIT differential emission measure (DEM) analysis (Cook et al. 1999, 2004). Since there are numerous individual emission lines formed over a range of temperatures in each EIT bandpass, it is difficult to interpret the images in a temperature sense. For example, the $195 \AA$ bandpass includes tens of lines from Fe $\mathrm{X}$ to Fe XIII, and the $304 \AA$ bandpass includes, among others, the He II $303.78 \AA$ and the Si XI $303.324 \AA$ lines. However, a technique developed by Cook et al. $(1999,2004)$ based on DEM analysis makes it possible to recover the spectral information. From a set of four synoptic EIT images, a DEM curve is derived for each of the $1024 \times 1024$ pixels. An initial smooth DEM curve is modified so that the synthetic intensities best reproduce the EIT observations for each pixel.

From these one million DEM curves, called a DEM map, one can calculate (using the CHIANTI package, see Dere et al. 1997) synthetic intensity images of the corona in any spectral line in the temperature range covered by the EIT, i.e. from $80000 \mathrm{~K}$ for He II $304 \AA$ to $2 \mathrm{MK}$ for Fe XV $284 \AA$. Including the $10 \%$ uncertainties in the radiometric calibration of the EIT (Newmark et al. 2004), this technique has shown to provide intensities accurate to within $20 \%$, and the results have been compared to simultaneous measurements provided by the CDS and CELIAS/SEM onboard SOHO, see McMullin et al. (2002) and references therein. The EIT $304 \AA$ channel presents a special problem for the DEM technique because the He II $303.78 \AA$ line, which is the main contributor to this channel, is optically thick. The intensity of this line calculated using standard DEM curves is much less than the observed intensity. Fortunately, there are excellent observations of both He II $303.78 \AA$ line intensity and the intensity ratio of this line to the nearby optically thin Si XI line at $303.324 \AA$. The DEM technique uses an empirical intensity multiplier for the He II line that reproduces observed quiet and active region intensities, and also the observed intensity ratio to the $\mathrm{Si} \mathrm{XI}$ line.

A full EIT synoptic set takes about 20 minutes to complete. The DEM analysis thus makes sense only if the corona is believed not to have changed dramatically during this period of time. The technique can therefore be applied to synoptic sets taken before and/or after a CME, but does not allow the investigation of the dynamic phase.

Fortunately, the synoptic set in the four EIT bandpasses was made just after the event (07:00-07:18 UT). Since the previous synoptic set (01:00-01:18 UT) until the start of the event (04:34 UT) the corona did not exhibit significant changes. Thus we assume that the plausible approximation of the pre-event atmosphere would be the images (and DEM map) taken during the synoptic set around 01:00 UT rotated to the time of postevent synoptic set (we adopted 07:12 UT).

To present examples of DEM distributions we have selected two typical pixels as shown in Fig. 5. After the eruption the pixel 1 is situated inside a TCH; the pixel 2 - outside TCHs but inside the overall dimming area. Pre-event and post-event DEM curves for these two pixels are presented in Fig. 6. From the decrease of the DEM, it is clear that the matter has been erupted from inside the TCHs (Fig. 6, left panel) as well as from

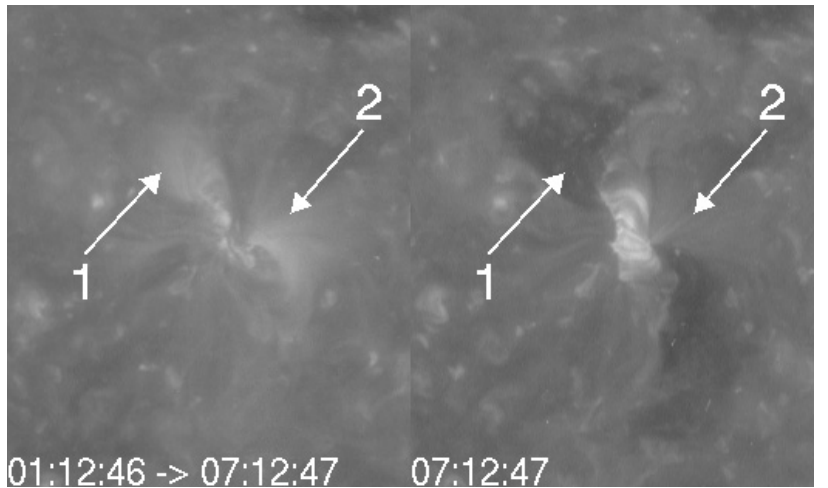

Fig. 5. Area around NOAA AR 8038 observed by the SOHO/EIT in the Fe XII bandpass (195 $\AA$ ) on May 12, 1997 at 01:12:46 UT (left panel) and 07:12:47 UT (right panel). The image made at 01:12:46 UT is rotated to 07:12:47 UT. Arrows 1 and 2 indicate the pixels for which DEM curves are plotted in Fig. 6.

outside of them (Fig. 6, right panel), although the emission measure decrease is stronger inside the TCHs. These curves are just illustrative examples; the mass estimates were made using individual DEM curves for each pixel. On average over the whole dimming area, the DEM decrease at $\log T<5.3$ is negligible.

The area of TCHs can be determined using brightness segmentation method (see e. g. Veselovsky et al. 2001; Zhukov et al. 2002). As for usual coronal holes in the $195 \AA$ bandpass, we assume that at this phase of the solar cycle pixels with brightness $I \leq 45 \mathrm{DN} \mathrm{s}^{-1}$ are situated inside TCHs (brightness is expressed in units of digital numbers per second in fully calibrated images). The overall dimming area is determined as pixels with negative brightness difference between the post-eruption 07:12 UT image and the rotated pre-eruption 01:12 UT image (both in $195 \AA$ ). It appears that our method of CME mass calculation is not too sensitive to the TCH boundaries position, to the dimming area determination (using the difference in the Fe XII brightness or in the emission measure) and to the exact value of the pressure scale height.

To determine the EM, we have integrated the individual DEM curves for each pixel inside the TCHs and inside the larger dimming over temperatures between $\log T=5.0$ and $\log T=6.5$. We did not include the cool low transition region $(\log T<5.0)$ in our computation. The DEM calculation at low temperatures relies only on the He II bandpass of the EIT. The main line in this bandpass - He II at $303.78 \AA$ with $\log T_{\max }=4.9-$ is optically thick, so the opacity effects come into play. The He II bandpass (304 $\AA$ ) does not exhibit pronounced TCHs and shows only weak dimmings (see Fig. 4).

Our calculations show that the mass removed from the low corona during this CME event is more or less equally distributed between the compact dark TCHs and the more extended weak dimming. We found that approximately $7 \times 10^{14} \mathrm{~g}$ erupts from the TCHs, and $1.4 \times 10^{15} \mathrm{~g}$ erupts from the whole dimming region (including TCHs). The mass of the halo CME associated with the described event can be estimated on the basis of white-light LASCO data. It should be noted, however, that such an estimate depends on the position of the CME with 

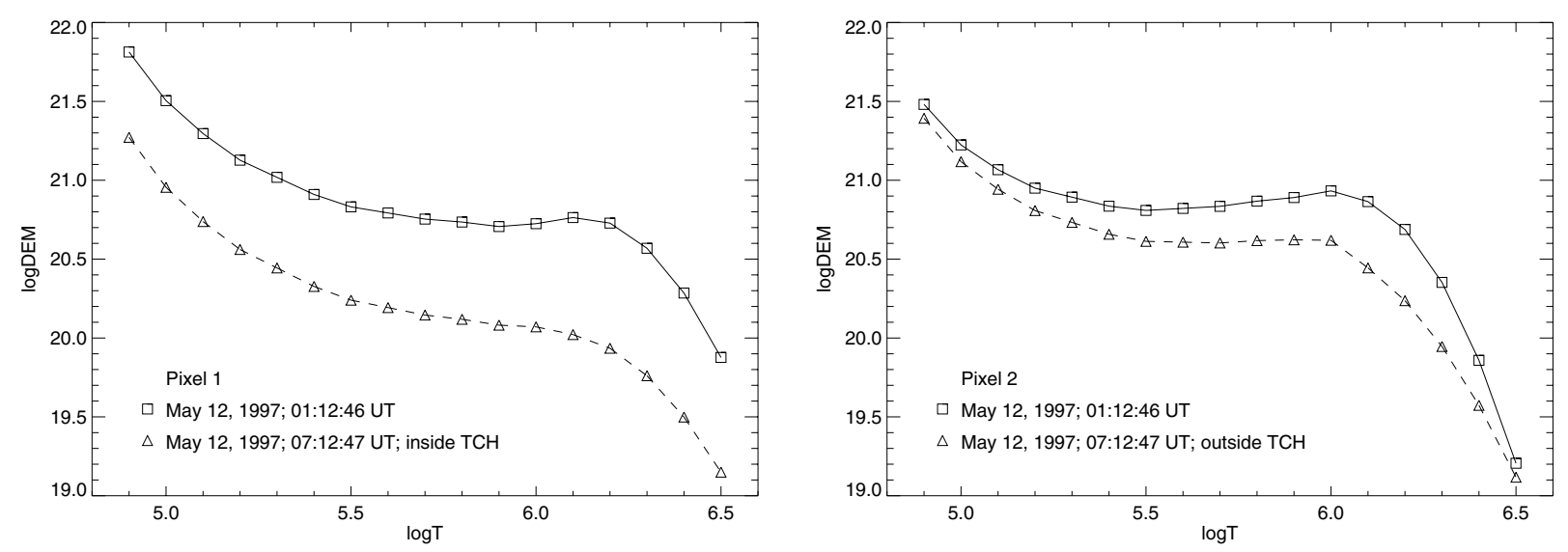

Fig. 6. Differential emission measure (DEM, in $\mathrm{cm}^{-5} \mathrm{~K}^{-1}$, logarithmic scale) curves for pixels 1 (left panel) and 2 (right panel) shown in Fig. 5. Pixel 1 corresponds to a typical pixel that occurred to be inside a TCH after the eruption; pixel 2 - outside the TCHs but inside the main dimming area. Squares and solid curve represent the pre-eruption DEM (01:12:46 UT rotated to 07:12:47 UT); triangles and dashed curve the post-eruption DEM (07:12:47 UT).

respect to the sky plane, which is not known. Assuming that the CME material is close to the plane of the sky, one can obtain the lower limit of the true CME mass: it is $4.2 \times 10^{15} \mathrm{~g}$ (Vourlidas 2004). This value falls inside the typical white-light CME mass range of $2 \times 10^{14} \mathrm{~g}$ to $4 \times 10^{16} \mathrm{~g}$ (Howard et al. 1985; Hundhausen 1997). Taking into account that our calculations provide only rough order of magnitude estimates, there is a fair agreement with our value.

We also compare our estimate of a CME mass with those obtained using other observations of the low corona. Sterling \& Hudson (1997) used a similar method to calculate the mass on the basis of Yohkoh/SXT emission measure changes. They obtained a few times $10^{14} \mathrm{~g}$ for the mass evacuated from the transient coronal holes for a CME on April 7, 1997. Harrison \& Lyons (2000) and Harrison et al. (2003) analyzed the dimmings on the limb observed in different spectral lines by the $\mathrm{SOHO} / \mathrm{CDS}$ and obtained CME masses in the range of $4 \times 10^{13} \mathrm{~g}-10^{17} \mathrm{~g}$. We see that our values $\left(7 \times 10^{14} \mathrm{~g}\right.$ erupted from TCHs and $1.4 \times 10^{15} \mathrm{~g}$ overall) are in good agreement with other estimates of CME mass.

\section{Discussion and conclusions}

We reported several examples of EIT waves and EUV dimmings with interesting and particular morphologies. We hope that the analysis of these events can shed additional light on the still-enigmatic process of CME onset.

The February 9, 1999 event is a rare example of a CME initiation observed in the Fe XV (284 $\AA$ ) bandpass of the SOHO/EIT. It confirms that EIT waves are coronal phenomena as they are observed in the coronal EUV emission. It is still unclear if EIT waves can be observed in the transition region (TR) lines. Modern instruments do not show any definite EIT wave signatures in the transition region bandpasses (the interpretation of lower TR observations in He II and also He I is ambiguous). Thus the association of EIT waves with the chromospheric Moreton waves is still questionable. If these waves represent the same phenomenon, one can expect that the wave should be observed also between the corona and the chromosphere, i.e. in the TR.

Can EIT waves be regarded as decelerated Moreton waves - when the wave positions lie on the same kinematic curve approximated by a parabolic fit (see Warmuth et al. 2001, 2004a)? The observations reported by Eto et al. (2002) exclude such a possibility at least for the event described in their work. More simultaneous observations of EIT and Moreton waves are needed. Moreover, the interpretation of Warmuth et al. (2001, $2004 a, b)$ is valid only assuming high values of the coronal plasma parameter $\beta>1$. So, there is increasing evidence that Moreton and EIT waves are two distinct phenomena.

Similarly, the number of EIT waves sometimes observed in SXR ("X-ray waves") is not yet sufficient to draw conclusions about their association with "true" EIT waves (observed by the EIT in the Fe XII bandpass). Yohkoh/SXT observations usually give speeds of the order of $500 \mathrm{~km} \mathrm{~s}^{-1}$, but recent observations by GOES/SXI registered values between 50 and $350 \mathrm{~km} \mathrm{~s}^{-1}$, which are close to the classical EIT wave speed $\left(250 \mathrm{~km} \mathrm{~s}^{-1}\right)$.

A serious problem now is the EIT waves undersampling in time. With typical EIT "CME Watch" cadence of about 12 min the wave front can be seen only in 3-4 images. This is not enough for the precise determination of the starting time and of the acceleration. Moreover, the EIT wave front in the first registered image is often observed far enough from the eruption site where the Moreton wave is no longer visible. The TRACE cadence is good enough for investigations of EIT waves, but its small field of view and automatic exposure control during flares make the observations of EIT waves by TRACE rare. Future missions like the Solar Dynamics Observatory (SDO) should provide simultaneous high-cadence (up to $10 \mathrm{~s}$ ) observations of EIT waves in several EUV bandpasses.

It is still not clear if EIT waves represent real MHD waves or if they rather result from successive opening of the magnetic field lines. We suppose that observations of EIT waves close to the solar limb (like the two examples described in Sect. 3) may provide important information concerning this question. On the basis of these observations we suggest that an EIT wave can be regarded as a bimodal phenomenon. The wave mode 
is a wave-like propagating disturbance and represents the pure MHD wave (the fast magnetosonic one in a high- $\beta$ plasma?) that may or may not steepen to form a shock. The characteristic features of the wave mode are: propagation of a bright front to large distances from dimming sites; quasi-circular appearance of the bright front. The eruptive mode is the propagation of the bright front (compression) and a dimming as a result of successive opening of magnetic field lines during the CME lift-off. The eruptive mode can be identified by noting the expansion of a dimming and the appearance of another dimming ahead of a bright front. Both modes are probably present in any EIT wave event (but not necessarily detected by modern instruments).

The eruptive mode of EIT waves has been modeled by Chen et al. (2002). The nature of the wave mode is, however, still unclear. Is the wave mode of an EIT wave indeed an MHD wave? On the one hand, the velocity of the wave mode is typically about $250 \mathrm{~km} \mathrm{~s}^{-1}$, and it is too low to be the fast magnetosonic wave speed in a low- $\beta$ coronal plasma. On the other hand, the wave mode often propagates rather isotropically from the eruption site (e.g. event of May 12, 1997) and thus cannot be a slow magnetosonic wave with a speed depending on the orientation of the wave vector with respect to the magnetic field. The isotropic propagation does not allow us to interprete all EIT waves in terms of the eruptive mode. It is not easy to imagine how the supposed magnetic configuration of a bipolar arcade can produce such an isotropically propagating bright front. Moreover, EIT waves can propagate to large distances from the dimming area. Therefore, the question about the physical nature of the wave mode of EIT waves is still open.

Transient coronal holes often represent only part of the overall dimming and thus of the overall region from which the mass is evacuated. The DEM calculations on the basis of EIT images permit us to estimate the mass removed from the low corona during the CME event on May 12, 1997 (classical EIT wave example). It is around $10^{15} \mathrm{~g}$, and only half of this total mass is removed from the TCH locations. This is the first estimate of a CME mass made using EIT images and a DEM analysis. Our value agrees well with previous typical CME mass estimates.

Acknowledgements. We are greatly indebted to J. Cook and J. Newmark for kindly providing us with the EIT DEM calculation software. EIT and LASCO data have been used courtesy of $\mathrm{SOHO} / \mathrm{EIT}$ and SOHO/LASCO consortiums. SOHO is a project of international cooperation between ESA and NASA. We also thank D. Berghmans for useful discussions.

\section{References}

Biesecker, D. A. 2003, private communication

Biesecker, D. A., Myers, D. C., Thompson, B. J., Hammer, D. M., \& Vourlidas, A. 2002, ApJ, 569, 1009

Chen, P. F., Wu, S. T., Shibata, K., \& Fang, C. 2002, ApJ, 572, L99

Chertok, I. M., \& Grechnev, V. V. 2003, in Proceedings of ISCS 2003: Solar Variability as an Input to the Earth's Environment, ESA SP-535, 435

Cook, J., Newmark, J., \& Moses, D. 2004, in preparation
Cook, J. W., Newmark, J. S., \& Moses, J. D. 1999, in Proceedings 8th SOHO Workshop: Plasma Dynamics and Diagnostics in the Solar Transition Region and Corona, ESA SP-446, 241

Del Zanna, G., Bromage, B. J. I., \& Mason, H. E. 2003, A\&A, 398, 743

Delaboudinière, J.-P., Artzner, G. E., Brunaud, J., et al. 1995, Sol. Phys., 162, 291

Delannée, C. 2000, ApJ, 545, 512

Delannée, C., \& Aulanier, G. 1999, Sol. Phys., 190, 107

Delannée, C. 1999, in Proceedings 8th SOHO Workshop: Plasma Dynamics and Diagnostics in the Solar Transition Region and Corona, ESA SP-446, 275

Dere, K. P., Landi, E., Mason, H. E., Monsignori Fossi, B. C., \& Young, P. R. 1997, A\&AS, 125, 149

Eto, S., Isobe, H., Narukage, N., et al. 2002, PASJ, 54, 481

Gilbert, H. R., Holzer, T. E., Thompson, B. J., \& Burkepile, J. T. 2004 , ApJ, 607, 540

Handy, B. N., Acton, L. W., Kankelborg, C. C., et al. 1999, Sol. Phys., 187,229

Harra, L. K., \& Sterling, A. C. 2001, ApJ, 561, L215

Harrison, R. A., Bryans, P., Simnett, G. M., \& Lyons, M. 2003, A\&A, 400, 1071

Harrison, R. A., \& Lyons, M. 2000, A\&A, 358, 1097

Howard, R. A., Sheeley, N. R., Michels, D. J., \& Koomen, M. J. 1985 , J. Geophys. Res., 90, 8173

Hudson, H. S., Khan, J. I., Lemen, J. R., Nitta, N. V., \& Uchida, Y. 2003, Sol. Phys., 212, 121

Hundhausen, A. J. 1997, in Coronal Mass Ejections, ed. N. Crooker, J. Joselyn, \& J. Feynman, AGU Geophys. Monograph. Ser., 212, 1 Kahler, S. W., \& Hudson, H. S. 2001, J. Geophys. Res., 106, 29239

Khan, J. I., \& Aurass, H. 2002, A\&A, 383, 1018

McMullin, D. R., Woods, T. N., Dammasch, I. E., et al. 2002, in The Radiometric Calibration of SOHO, ISSI Scientific Report SR-002, ed. A. Pauluhn, M. C. E. Huber, \& R. von Steiger, 317

Moreton, G. E. 1960, AJ, 65, 494

Moreton, G. E., \& Ramsey, H. E. 1960, PASP, 72, 357

Narukage, N., Hudson, H. S., Morimoto, T., et al. 2002, ApJ, 572, L109

Newmark, J. S., Cook, J. W., Moses, J. D., Auchère, F., \& Clette, F. 2004, Sol. Phys., to be submitted

Plunkett, S. P., Thompson, B. J., Howard, R. A., et al. 1998, Geophys. Res. Lett., 25, 2477

Schmelz, J. T., Beene, J. E., Nasraoui, K., et al. 2003, ApJ, 599, 604

Sterling, A. C., \& Hudson, H. S. 1997, ApJ, 491, L55

Thompson, B. J., Cliver, E. W., Nitta, N., Delannée, C., \& Delaboudinière, J. P. 2000a, Geophys. Res. Lett., 27, 1431

Thompson, B. J., Gurman, J. B., Neupert, W. M., et al. 1999, ApJ, 517, L151

Thompson, B. J., Plunkett, S. P., Gurman, J. B., et al. 1998, Geophys. Res. Lett., 25, 2465

Thompson, B. J., Reynolds, B., Aurass, H., et al. 2000b, Sol. Phys., 193, 161

Uchida, Y. 1968, Sol. Phys., 4, 30

Uchida, Y. 1974, Sol. Phys., 39, 431

Uchida, Y., Altschuler, M. D., \& Newkirk, G. J. 1973, Sol. Phys., 28, 495

Veselovsky, I. S., Zhukov, A. N., Dmitriev, A. V., et al. 2001, Sol. Phys., 201, 27

Vourlidas, A. 2004, private communication

Vršnak, B., Warmuth, A., Brajša, R., \& Hanslmeier, A. 2002, A\&A, 394, 299

Wang, T., Yan, Y., Wang, J., Kurokawa, H., \& Shibata, K. 2002, ApJ, 572,580 
Wang, Y.-M. 2000, ApJ, 543, L89

Warmuth, A., Vršnak, B., Aurass, H., \& Hanslmeier, A. 2001, ApJ, 560, L105

Warmuth, A., Vršnak, B., Aurass, H., \& Hanslmeier, A. 2002, in SOLSPA 2001: Proceedings of the Second Solar Cycle and Space Weather Euroconference, ESA SP-477, 195

Warmuth, A., Vršnak, B., \& Hanslmeier, A. 2003, Hvar Observatory Bulletin, 27, 139

Warmuth, A., Vršnak, B., Magdalenić, J., Hanslmeier, A., \& Otruba, W. 2004a, A\&A, 418, 1101
Warmuth, A., Vršnak, B., Magdalenić, J., Hanslmeier, A., \& Otruba, W. 2004b, A\&A, 418, 1117

Webb, D. F., Lepping, R. P., Burlaga, L. F., et al. 2000, J. Geophys. Res., 105, 27251

Wills-Davey, M. J., \& Thompson, B. J. 1999, Sol. Phys., 190, 467 Wu, S. T., Zheng, H., Wang, S., et al. 2001, J. Geophys. Res., 106, 25089

Zhukov, A. N., Veselovsky, I. S., Hochedez, J.-F., et al. 2002, in Proceedings of the SOHO 11 Symposium: From Solar Min to Max: Half a Solar Cycle with SOHO, ESA SP-508, 189 